\title{
The Freedoms of Guarded Bisimulation
}

\author{
Martin Otto \\ Department of Mathematics \\ Technische Universität Darmstadt \\ otto@mathematik.tu-darmstadt.de
}

\begin{abstract}
Guarded logics have been shown to be amazingly versatile and tractable logics since their inception by Andréka, van Benthem, Németi in [1]. Features otherwise known from modal logics are lifted to the richer setting of general relational structures. The leading idea in this generalisation is the relativisation of quantifiers to tuples that are guarded by relational atoms. In a sense that can be made precise for many specific issues, guarded logics relate to general relational structures and hypergraphs in much the same way that modal logics relate to Kripke structures and graphs. This is particularly apparent for the associated semantic equivalence games. Guarded bisimulation can be seen as derived from a hypergraph version of ordinary (modal) bisimulation for graph-like structures. Just like preservation under ordinary bisimulation accounts for much of the good model-theoretic behaviour of modal logics, so hypergraph bisimulation and guarded bisimulation are the keys to understanding the model theory of guarded logics [1, 3, 4]. Model constructions and transformations that are compatible with guarded bisimulation account for the malleability of models and the tractability of the finite and algorithmic model theory of various guarded logics. They can often be cast in terms of hypergraph constructions [5, 6]. Here I intend to survey and summarise a number of results in the light of such model constructions including some more recent developments from $[2,6]$. Results to be surveyed include finite and small model properties, decidability results, complexity and expressive completeness issues. At the conceptual as well as at the technical level, guarded bisimulations and local versus global properties in the overlap patterns of (finite) hypergraph structures form one of the leading themes, and offer intriguing combinatorial challenges.
\end{abstract}

1998 ACM Subject Classification F.4.1 Mathematical Logic

Keywords and phrases Model theory, guarded logic, bisimulation, hypergraphs

Digital Object Identifier 10.4230/LIPIcs.CSL.2011.2

\section{References}

1 H. Andréka, J. van Benthem and I. Németi, Modal languages and bounded fragments of predicate logic, Journal of Philosophical Logic, 27 (1998), pp. 217-274.

2 V. Barany and G. Gottlob and M. Otto, Querying the guarded fragment, Proc. 25th IEEE Symposium on Logic in Computer Science, 2010, pp. 1-11. Preprint of journal version (submitted to LMCS) available online.

3 E. Grädel, On the restraining power of guards, Journal of Symbolic Logic, 64 (1999), pp. $1719-1742$.

4 E. Grädel, C. Hirsch and M. Otto, Back and forth between guarded and modal logics, ACM Transactions on Computational Logic, 3 (2002), pp. 418-463.

5 I. Hodkinson and M. Otto, Finite conformal hypergraph covers and Gaifman cliques in finite structures, The Bulletin of Symbolic Logic, 9 (2003), pp. 387-405.

6 M. Otto, Highly acyclic groups, hypergraph covers and the guarded fragment, Proc. 25th IEEE Symposium on Logic in Computer Science, 2010, pp. 12-21. Preprint of journal version (to appear in JACM) available online. 